\title{
ENCYCLIA CAJALBANENSIS (ORCHIDACEAE), UNA ESPECIE NUEVA DE LA FLORA CUBANA
}

\author{
Ernesto Mujica Benitez ${ }^{1,3}$, José L. Bocourt Vigil ${ }^{1} \&$ Franco Pupulin ${ }^{2}$ \\ 'Orquideario Soroa, Universidad de Pinar del Río, Apdo. postal No. 5 Candelaria, Pinar del Río, Cuba \\ ${ }^{2}$ Jardín Botánico Lankester, Universidad de Costa Rica, apdo. 1031-7050 Cartago, Costa Rica. \\ Investigador asociado de Marie Selby Botanical Gardens, Florida, EE.UU.
}

${ }^{3}$ Autor para correspondencia: inv@orq.pr.minaz.cu

\begin{abstract}
A new species of the genus Encyclia, endemic to the Cajálbana plateau in Cuba, is described and illustrated. Encyclia cajalbanensis is similar to E. fucata, from which it differs by the greater size of the plant and the much larger lip, provided with a reniform-suborbicular midlobe.

RESUMEN. Se describe e ilustrra una nueva especie de Encyclia, endémica de la meseta de Cajálbana en Cuba. Encyclia cajalbanensis es parecida a E. fucata, de la cual se diferencia por el mayor tamaño de las plantas y por el labelo mucho más grande, con el lóbulo medio reniforme, suborbicular.
\end{abstract}

Palabras clave / Key words: Orchidaceae, Encyclia cajalbanensis, Encyclia fucata, Cuba

Encyclia Hook. es un género neotropical con unas 140 especies, de las cuales se registran unas 20 a 25 especies en el Caribe (Withner 1996, Nir 2000, Ackerman, com. pers., 2004). La isla de Cuba presenta la mayor diversidad del género en la región antillana, pero el cálculo del número de táxones ha sido controversial. En sus tratamientos de Epidendrum para la flora cubana, Acuña (1938) y el Hermano León (Sauget y Barbier 1946) anotan 19 especies referibles a Encyclia s.s. (con siete especies endémicas). Muchos de estos nombres fueron posteriormente tratados como sinónimos y hoy se consideran informes erróneos en la flora de Cuba. Dietrich (1984) registra 22 especies de Encyclia, un número que refleja los conceptos específicos muy estrechos utilizados por esta botánica alemana, así como la inclusión en su lista de nombres aportados por varios autores, que no fueron documentados por testigos cubanos verificables. En su revisión de las especies antillanas, Withner (1996) registra 11 especies de Cuba, de las cuales más que la mitad se indican como endémicas [E. howardii (Ames \& Correll) Hoehne, E. ochrantha (A.Richard) Withner, E. oxypetala (Lindl.) Schltr., E. phoenicea (Lindl.) Neum., E. pyriformis (Lindl.) Schltr. y E. triangulifera (Rchb.f.) Acuña]. En el catálogo de orquídeas cubanas publicado por
Mújica y colaboradores, el número de especies de Encyclia se acerca al registrado por Acuña y el Hermano León; son 17 especies (Mújica et al. 2000). En su reciente monografía de las orquídeas antillanas, Nir (2000) reconoció 13 especies de Encyclia en la flora cubana, con seis táxones considerados como endémicos. En su tratamiento de la familia Orchidaceae para la flora de las Antillas Mayores, Ackerman (en prep.) reconoce en la flora de Cuba un total de 15 especies, con un alto índice de endemismo, pues 10 especies son exclusivas de la isla. Actualmente, y de acuerdo con los últimos estudios realizados por el primer autor, el número de especies cubanas podría sobrepasar las 20 , teniendo en cuenta la existencia del "complejo E. phoenicea", cuyo estudio podría dar como resultado la segregación de nuevos táxones dentro del género (Mújica et al., en prep.).

Durante los trabajos de campo efectuados en la provincia de Pinar del Río, específicamente en la meseta de Cajálbana, se recolectaron varios ejemplares de este género. Como resultado de los trabajos preliminares de revisión del género Encyclia para una monografía de las especies cubanas (Mújica et al., en prep.), el presente artículo quiere dar a conocer una especie nueva para la ciencia, endémica de Cuba. 


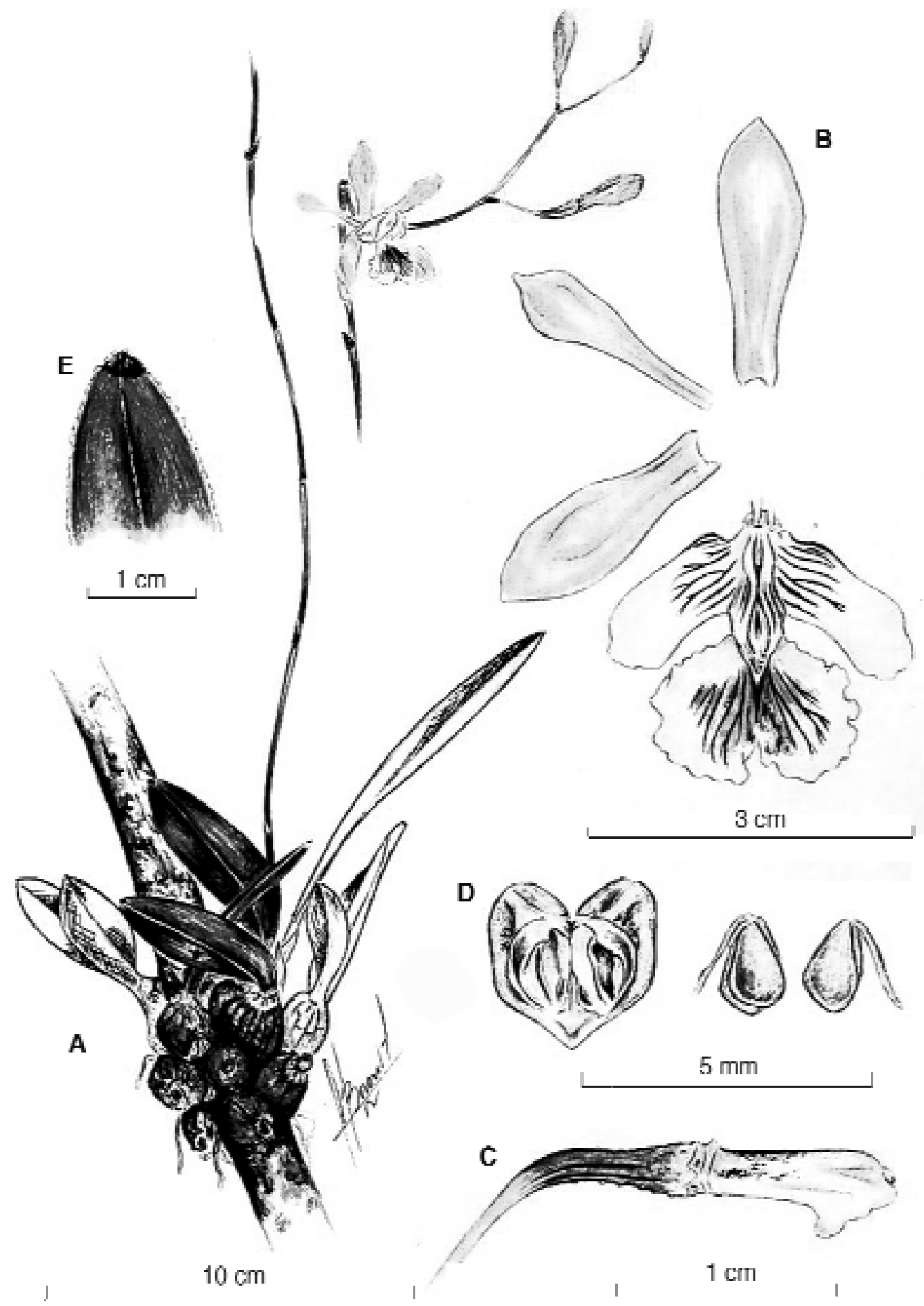

Figura 1. Encyclia cajalbanensis Mújica, Bocourt \& Pupulin. A. Hábito, B. Perianto seccionado, C. Columna, vista lateral, D. Antera y polinarios, E. Ápice de la hoja. Dibujos del holotipo por J.L. Bocourt Vigil.. 
Encyclia cajalbanensis Mújica, Bocourt \& Pupulin, sp. nova

TIPO: Cuba. Pinar del Río; Municipio La Palma, ladera sur de la meseta de Cajálbana, a $2.5 \mathrm{~km}$ después de El Burén, en Sendero Interpretativo, Mil Cumbres, en cuabales [bosques esclerófilos secos], floreció en cultivo en el Orquideario Soroa en junio de 2002, J.L. Bocourt 117 (Holotipo: HOS, Herbario del Orquideario Soroa, Pinar del Río, Cuba; isotipo: flores en alcohol, HOS-035).

FIG. 1.

Species Encycliae fucatae (Lindl.) Britt. et Millsp. similis, sed planta habitu majore, labello valde majore cum lobulo medio reniformi-suborbiculari recedit.

Epífita o litófita de hasta $45 \mathrm{~cm}$ de altura en floración. Raíces numerosas, delgadas. Rizoma muy corto, ascendente. Pseudobulbos muy agregados, ovoides, corrugados, de unos 2-4 cm de largo. Hojas $1-2$, carnosas, muy rígidas, conduplicadas en la base, oblongo-lanceoladas, agudas, con margen ligeramente dentado, muy variables en tamaño, de $2-15 \mathrm{~cm}$ de largo y $1,5 \mathrm{~cm}$ de ancho. Inflorescencia simple, un racimo erecto y delgado de hasta $60 \mathrm{~cm}$ de altura, con una bráctea basal, abrazadora, en el escapo floral, flores hasta 15 , con brácteas que se van espaciando hacia el ápice. Brácteas florales pequeñas, abrazadoras, triangulares, agudas, de $2 \mathrm{~mm}$ de longitud. Ovario verrugoso, de $14 \mathrm{~mm}$ de largo (con el pedice1o). Flores vistosas, verde amarillentas, con labelo blanco y venación púrpura, con aroma agradable perceptible. Sépalos oblanceolado-espatulados, agudos, el dorsal de 12-15 mm de largo $\times 5 \mathrm{~mm}$ de ancho, los laterales de 12-14 $\mathrm{mm}$ de largo $\times 4 \mathrm{~mm}$ de ancho. Pétalos espatulados, agudos, similares en tamaño a los sépalos laterales. Labelo libre, extendido, trilobulado, lóbulos laterales erectos, abrazando la columna, oblongos, redondeados apicalmente, con estrías púrpuras, de ca. $1 \mathrm{~cm}$ de largo x 4-5 mm de ancho, lóbulo medio reniforme-suborbicular, emarginado, con márgenes ondulados y venación púrpura, de 10-12 mm de largo a partir del istmo y $12-15 \mathrm{~mm}$ de ancho, disco bilamelado, fusionándose ambas quillas en el ápice. Columna alada, robusta, dorsalmente con manchas púrpuras hacia la base, de $8 \mathrm{~mm}$ de longitud. Antera aovada, amarillenta, de $2 \mathrm{~mm}$ de ancho. Polinios 4 , obpiriformes, cerosos, en dos pares, de 1,5 $\mathrm{mm}$ de longitud, en dos caudículas bífidas.

Etimología. El epíteto cajalbanensis se dedica al lugar de origen, la Meseta de Cajálbana, área rica en especies endémicas.

ECOlOGÍA Y Distribución. Abundante en los cuabales de la ladera sur de la meseta de Cajálbana, creciendo sobre los arbustos o directamente en el suelo, sobre las formaciones rocosas del lugar. Florece entre junio y julio.

Estatus de CONSERVACión. Esta es la única población conocida y forma parte de una reserva natural, por lo que actualmente no existen factores de riesgo, que pongan en peligro su existencia y su propagación natural.

Esta especie, al parecer, era confundida con E. fucata, de la cual se diferencia por su mayor tamaño y por la morfología de su labelo, que es mucho más grande. El hábitat de esta especie es bien diferente al que prefiere E. fucata. Mientras la primera habita en vegetación de cuabal, la segunda se encuentra por lo general en bosques húmedos y perennifolios.

Agradecimientos. Los autores agradecen a James D. Ackerman, de la Universidad de Puerto Rico, por la revisión del texto, sus opiniones y su apoyo en todo momento.

\section{LiTERATURA CITADA}

Ackerman, J.D. 1995. An Orchid Flora of Puerto Rico and the Virgin Islands. Mem. New York Bot. Gard. 73. Bronx. New York.

Acuña, J. 1938. Catálogo descriptivo de las orquídeas cubanas. Bol. Est. Agron. Santiago de las Vegas 60.

Dressler, R.L. 1974. El género Encyclia en México. Asociación Mexicana de Orquideología. México.

Mújica, E.M. (y colaboradores). 2000. Los Géneros de Orquídeas Cubanas. Editorial Félix Varela. La Habana.

Nir, M.A. 2000. Orchidaceae Antillanae. DAG Media Publishing, Inc. New York.

Sauget y Barbier, J.S. [Hermano León]. 1946. Flora de Cuba, vol. 1. Contr. Oc. Mus. Hist. Nat. Col. La Salle 8: 373-382.

Withner, C.L. 1996. The Cattleyas and their Relatives. 4. The Bahamian and Caribbean Species. Timber Press. Portland. Oregon. 